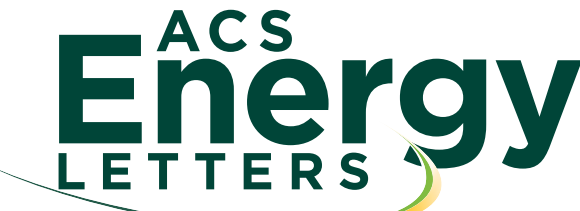

\section{Impedance Spectroscopy Measurements in Perovskite Solar Cells: Device Stability and Noise Reduction}

$\mathrm{P}$ erovskite solar cells (PSCs) are the most recent newcomers to the photovoltaic field and have attracted huge interest in the past few years due to their high photoconversion efficiencies (higher than 22\%) ${ }^{1}$ and their relatively easy fabrication process. $^{2}$ Despite that PSCs constitute a category of solar cells with its own peculiarities, earlier reports used perovskites to sensitize wide band gap semiconductors. ${ }^{3,4}$ Undoubtedly, the rapid increase in efficiency experienced by PSCs has much to be thankful for with all of the previous work done on low-temperature processed technologies as sensitized cells or organic photovoltaics. In this sense, it is not surprising to observe that researchers use most of the characterization techniques developed for these former technologies in order to characterize PSCs. However, translation of characterization methods has to take into account the specificities of PSCs to avoid potentially misleading procedures and results. In the case of PSCs, the most archetypical example is the photoconversion efficiency determination through the current-potential $(J-V)$ curves that cannot be directly used due to the reported hysteresis. ${ }^{5}$ Another electrical technique extensively used for the characterization of photovoltaic devices, especially on sensitized and organic solar cells, is impedance spectroscopy (IS). ${ }^{6}$ This Viewpoint aims to clarify some general aspects of IS characterization, focusing on the special characteristic of PSCs in order to obtain reliable data that can allow a fair comparison between different devices.

IS is a nondestructive electrical characterization method that can be implemented coupled with device illumination in order to characterize the solar cells under working conditions. Usually, IS is a small-voltage perturbation method where a DC potential establishes the stationary condition of the device and a small AC signal at different frequencies is applied. Consequently, IS allows complete device analysis at the working conditions decoupling the different physical processes with different characteristic times (frequencies). ${ }^{6}$ This fact makes IS significantly appealing for characterization because, potentially, different physical processes can be analyzed by a single measurement. On the other hand, the experimental setup is simple and the measurements are easy to perform. However, to fully benefit from the IS potentiality, a complete model of the device, conventionally formulated in terms of an equivalent circuit in which each element has a physical meaning, is needed. While this model is well-known for other kinds of devices, ${ }^{6}$ it is not completely established for PSCs. Nevertheless, IS has been extensively used for the characterization of PSC,,$^{7-24}$ as some parts of the spectra have been identified as recombination resistance, geometrical capacitance, or accumulation capacitance, allowing a quantitative analysis. The interpretation of IS measurements for PSCs is beyond the scope of this Viewpoint that focus on the experimental setup, measurements, and special considerations for PSCs.

IS measurements need the accomplishment of three fundamental conditions: (i) causality; (ii) linearity; and (iii) stability. Causality indicates that the measured response is due exclusively to the applied stimulus. To this extent, it is necessary to screen the sample from external perturbations; the standard procedure is to perform the IS measurements inside of a Faraday cage. Connection wires also need an appropriate electrical shielding to reduce significantly the electrical noise. Moreover, IS is based on the linearization of voltage and current equations considering that small perturbations over the voltage produces linear variations over the current. ${ }^{6} \mathrm{AC}$ perturbation has to be high enough to produce a noise-free signal but small enough to ensure linearity. There is no universal rule, and the accomplishment of these conditions will depend on the sample and on the measurement conditions. A clear example is depicted in Figure 1a. While the measurement of a PSC at a DC bias, $V_{\text {appl }}$ of $0.2 \mathrm{~V}$ and AC perturbation of 50 $\mathrm{mV}$ can be realized with a good linear approach, the condition of linearity is not fulfilled for $V_{\text {appl }}=0.8 \mathrm{~V}$ at the same AC perturbations. The increase of $\mathrm{AC}$ perturbation produces a higher response and consequently a better signal/noise ratio. Figure $1 \mathrm{~b}$ shows the impedance pattern of the cell analyzed in Figure $1 \mathrm{a}$ at $V_{\text {appl }}=0.2 \mathrm{~V}$ and different $\mathrm{AC}$ perturbation. As linearity is preserved in a tolerable manner even for relatively large $\mathrm{AC}$ perturbation, the main effect of the increase of the AC perturbation is the positive reduction of the electrical noise. Conversely, the same analysis performed at $V_{\text {appl }}=0.8 \mathrm{~V}$ (see Figure 1c) shows that the augmentation of $\mathrm{AC}$ perturbation does not just reduce the noise but also alters the result of the measurement due to loss in linearity.

Electrical noise can be especially significant at low frequencies. Unlike in the case of other photovoltaic technologies such as $\mathrm{Si}$ solar cells, ${ }^{25}$ where no physical processes with long characteristic times (small frequencies) are present, for PSCs it is important to obtain noise-free measurements at low frequency as slow processes occur in PSCs. ${ }^{26}$ In fact, this part of the spectrum is important to determine recombination resistance ${ }^{8,9}$ or accumulation capacitance. $^{18,21}$ There are different ways to reduce the noise during the IS measurements: (i) the increase of AC while linearity is not disturbed, as we have already discussed, (ii) the increase of the integration time, and/or (iii) the increase of the number of integration cycles during the measurement. While the former has the drawback of altering the linearity, the two latter increase

Received: March 22, 2018

Accepted: March 28, 2018 

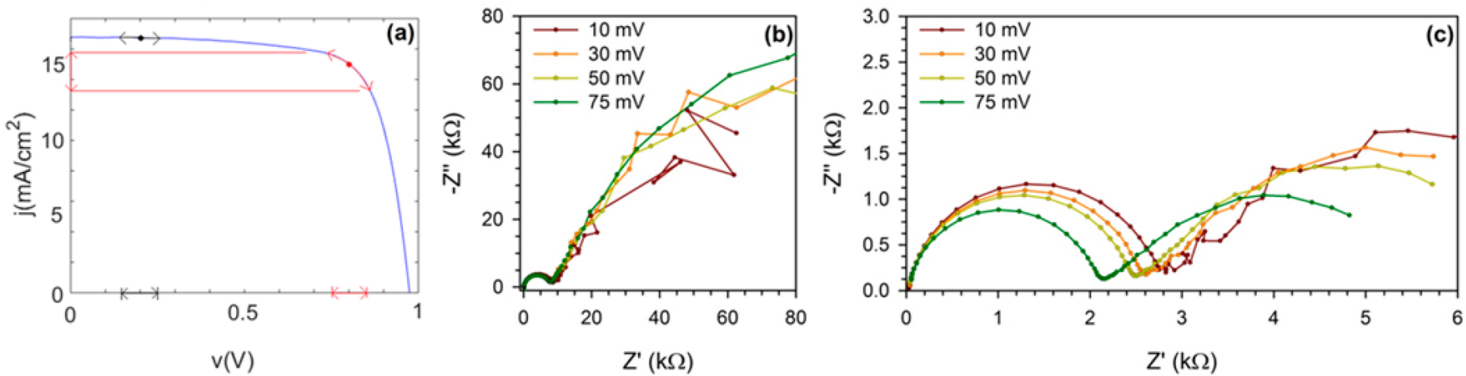

Figure 1. (a) $J-V$ curve of a PSC with $\mathrm{CH}_{3} \mathrm{NH}_{3} \mathrm{PbI}_{3}$ as the active layer. The variation of the current in terms of an modulated AC voltage of 50 $\mathrm{mV}$ is plotted for $V_{\text {appl }}=0.2$ and $0.8 \mathrm{~V}$. AC perturbation effect on the Nyquist plot for measurements of the same sample under 1 sun illumination, measured for a frequency range of $1 \mathrm{MHz}$ to $0.1 \mathrm{~Hz}$ with $V_{\text {appl }}=(\mathrm{b}) 0.2$ and (c) $0.8 \mathrm{~V}$. Details about PSCs fabrication can be found in the Supporting Information.
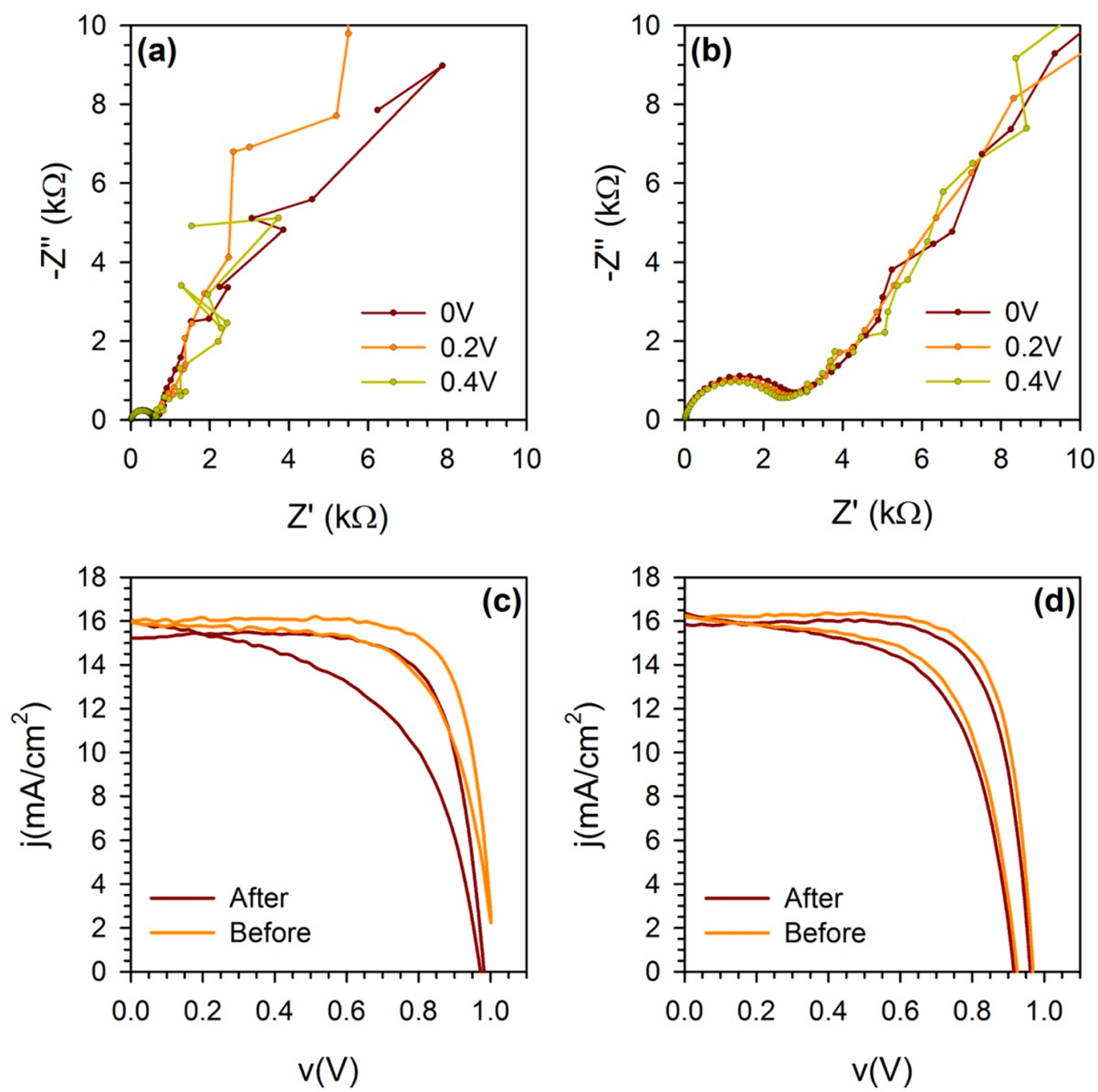

Figure 2. Nyquist plot at different $V_{\text {appl }}$ of the same PSC under (a) 1 sun and (b) 0.1 sun light intensity. $J-V$ curves with forward and reverse scans, corresponding to lower and higher apparent efficiencies, respectively, under 1 sun before and after the IS measurement at (c) 1 sun and (d) 0.1 sun light intensity.

the measurement time and could affect the third fundamental condition for IS measurement, the stability. As the scope of the IS measurement is to characterize the PSCs under stable working conditions, we suggest decreasing the measurement time as much as possible; consequently, the increment of integration time or the number of integration cycles is not advisible for low-stability samples. The use of dynamic AC perturbation, larger at low $V_{\text {appl }}$, where noise is higher and linearity easier to fulfill, and smaller close to the open-circuit voltage, $V_{\text {oo }}$ where the linearity condition is more demanding, is recommended; see Figure 1.

The major current challenge of PSCs is to increase their stability. There are multiple factors affecting the perovskite stability such as moisture, temperature, the nature of the 

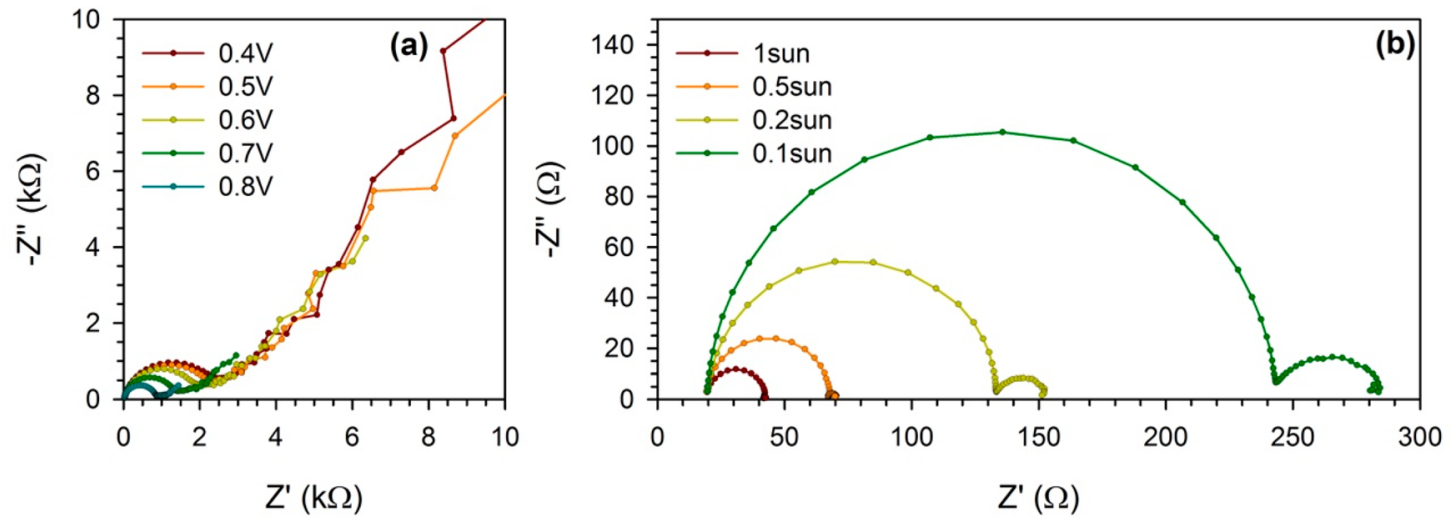

Figure 3. (a) Voltage scan under 0.1 sun and (b) light intensity scan at $V_{\mathrm{oc}}$, with 60 frequencies measured in the $1 \mathrm{MHz}-0.1 \mathrm{~Hz}$ frequency range in both cases.

selective contacts, the grain size, or light exposure. ${ }^{2}$ The stability of PSCs has increased significantly since their origin, and some perovskite configurations, such as as multiple cations, ${ }^{23,27}$ inorganic perovskites, ${ }^{22}$ or $2 \mathrm{D} / 3 \mathrm{D}$ layered perovskites, exhibit significant stability, ${ }^{28}$ even under illumination at the maximum power point. ${ }^{29,30}$ However, this Viewpoint aims to give a set of experimental procedures valid in the most general cases of PSCs, and long IS measurement can produce degradation of certain perovskite devices. Even if this process is enhanced under illumination, ${ }^{31}$ it is important to carry out the measurements of PSCs under illumination conditions as perovskite properties change dramatically under illumination. ${ }^{21,32}$ Although IS at 1 sun illumination conditions can be used to reconstruct the $J-V$ curve in the standard conditions for efficiency measurements, ${ }^{9,22}$ it is not necessary to carry out the IS measurements under 1 sun if IS aims at doing a comparative analysis between samples. For example, the analysis under 0.1 sun conditions, on one hand, brings the system to a high enough illumination to exhibit characteristic illumination features such as low-frequency accumulation capacitance ${ }^{18,21}$ and, on the other hand, reduces the electrical noise (compare panels (a) and (b) in Figure 2) and increases the device stability during measurement. The stability of the PSC can be quickly verified by comparing the $J-V$ curves before and after IS measurement (see Figure $2 \mathrm{c}, \mathrm{d}$ ). It can be appreciated that after IS at different applied voltages under 1 sun illumination the variation of the $J-V$ curve is significantly higher than that at 0.1 sun. It is important to highlight that the $J-V$ curve just after impedance measurement can produce a false impression of cell degradation due to the poling conditioning; we suggest measuring $J-V$ after different times to determine if the sample recovers its previous performance value (see Figure S1), allowing confidence in IS measurement as cell degradation is avoided.

There are several extrinsic procedures that contribute to increase the stability during the IS measurement, such as the use of a controlled temperature and atmosphere or encapsulation of the devices. In addition, there are also other intrinsic procedures for IS measurement conditions that can be used for this purpose that always have to be properly balanced to ensure the reliability of the measurement and the usefulness of the obtained data. A decrease of measurement time is beneficial in terms of stability, but it cannot be decreased by reducing excessively the low-frequency range, which is the most time-consuming, as important information is extracted from this range, as discussed above. We suggest using a frequency range starting at the highest frequency of the equipment setup and extending at least down to $0.1 \mathrm{~Hz}$. Of course, this frequency range could depend on the exact nature of each sample; for example, $0.1 \mathrm{~Hz}$ could be too high for a correct determination of DC resistance in samples with large lowfrequency resistance or presenting negative capacitance in the low or intermediate frequency range. ${ }^{71,22}$ An alternative is to reduce the total number of frequencies for a single $V_{\text {appl }}$; see Figures S2 and S3. In most cases, 40 frequencies distributed logarithmically in a frequency range between $1 \mathrm{MHz}$ and 0.1 $\mathrm{Hz}$ should be enough to obtain detailed impedance data.

Finally, it is important to avoid a practice unfortunately extended in the literature: the comparison of IS spectra of different samples at just a single condition, generally at $V_{\text {oc }}$. This kind of characterization does not allow a fair comparison because the Fermi level splitting is not the same in most cases. ${ }^{6}$ The carrier density depends exponentially on the Fermi levels. Meanwhile, important cell parameters such as the recombination rate, chemical capacitance, or conductivity depend on the carrier density. Different samples can present a shift of energy levels, causing, even at the same $V_{\text {app }}$, different conditions of Fermi level splitting to be reached. This fact is well-known in sensitized solar cells. ${ }^{6}$ Consequently, appropriate comparison has to be performed, sweeping the Fermi level splitting, either by fixing the illumination and applying different $V_{\text {appl }}$ (see Figure $3 \mathrm{a}$ ) or by using different illumination power densities, making a single spectrum at each illumination at the corresponding $V_{\text {oc }}$ (see Figure $3 \mathrm{~b}$ ). When illumination is fixed (Figure 3a), different applied biases between the short circuit and open circuit should be applied, while when illumination is changed it has to be varied over several orders of magnitude due to the exponential dependence between the Fermi level and the illumination power density. The latter could be more stressing from the stability point of view due to the spectra recorded at high illumination compared with a voltage scan at low illumination (see Figure 2) but can allow analysis of the ideality factor in parallel to IS measurements. ${ }^{33}$

In both cases, shown in Figure 3, a set of parameters at different Fermi level splitting can be obtained after the spectra fitting. One must note that when we speak about IS measurement we do not refer to obtaining a single impedance spectrum but refer to acquiring a complete set of spectra as in Figure 3. Depending on the experimental setup and settings, it will take $0.5-1 \mathrm{~h}$, being demanding conditions in terms of stability for certain PSCs devices. 
Table 1. Summary of the Common Problems Arising during the IS Measurement of Perovskites Solar Cells ${ }^{a}$

\begin{tabular}{|c|c|c|c|}
\hline problem & solution & drawback & good practice \\
\hline \multirow[t]{5}{*}{ noise (causality) } & Faraday cage & none & to do systematically \\
\hline & connection wire: electrical shielding & none & to do systematically \\
\hline & increase $\mathrm{AC}$ & possible linearity loss & adjust AC with $V_{\text {appl }}$ \\
\hline & increase integration time & increase the measurement time & reduce the number of frequency poin \\
\hline & $\begin{array}{l}\text { increase the number of integration } \\
\text { cycles }\end{array}$ & increase the measurement time & reduce the number of frequency poir \\
\hline \multirow[t]{3}{*}{$\begin{array}{l}\text { stability (cell evolution upon } \\
\text { measurement) }\end{array}$} & adjust the lowest frequency measured & $\begin{array}{l}\text { low-frequency part of the spectra must } \\
\text { remain analyzable }\end{array}$ & \\
\hline & $\begin{array}{l}\text { reduce the number of frequency } \\
\text { points/decade }\end{array}$ & the spectra must remain analyzable & $\begin{array}{l}\text {-follow } V_{\text {oc }} \text { after each measurement } \\
\text { (constant illumination) }\end{array}$ \\
\hline & $\begin{array}{l}\text { control temperature, use dry } \\
\text { atmosphere, encapsulate }\end{array}$ & none & $\begin{array}{l}\text {-measure } J-V \text { before and after IS at } \\
\text { different times }\end{array}$ \\
\hline stability and noise & reduce the illumination power density & for measurements at various $V_{\text {appl }}$ & \\
\hline
\end{tabular}

In summary, this Viewpoint provides some suggestions about good practices regarding IS measurements as to avoid the comparison of a single spectrum and compare a set of spectra at various Fermi level splittings by changing the applied bias or the illumination intensity. IS measurements have to ensure causality, linearity, and stability and at the same time provide low-noise useful data. Good shielding and performing measurements in a Faraday cage will contribute to warranty causality. Stability can be improved by controlling the measurement atmosphere and temperature by device encapsulation and also by reducing the measurement time. However, low-frequency data are important for appropriate characterization of PSCs, and as a consequence, the measurement time cannot be reduced dramatically. To that end, illumination conditions can be moderated and/or the number of total frequency points reduced. The noise of the measurement can be lowered with no further increase of measurement time by increasing the $\mathrm{AC}$ perturbation. The use of a dynamic AC stimulus larger at low applied voltage and lower at higher voltages can ensure the linearity conditions. The stability of the devices during the measurement has to be verified in order to be confident with the obtained data. The stability can be followed by recording the $V_{\text {oc }}$ between the measurements at various $V_{\text {appl }}$. Also measuring the $J-V$ curves before and after the impedance measurements can be a good proof of stability, knowing the recovery phenomenon that we have shown in this paper. Table 1 summarizes that careful IS measurements will allow one to obtain a set of reliable data for accurate PSC characterization.

Didac Pitarch-Tena ${ }^{\dagger}$

Thi Tuyen Ngo

Marta Vallés-Pelarda ${ }^{\dagger}$

Thierry Pauporté*,\$잉

Iván Mora-Seró* ${ }^{*} \dagger$

${ }^{\dagger}$ Institute of Advanced Materials (INAM), University Jaume I, Avenida de Vicent Sos Baynat, s/n, 12006 Castelló de la Plana, Castellón, Spain

${ }^{\ddagger}$ Institut de Recherche de Chimie-Paris (IRCP-UMR8247), CNRS-Chimie ParisTech-Paris Sciences et Lettres, 11 rue

Pierre et Marie Curie, 75005 Paris, France

\section{ASSOCIATED CONTENT}

\section{S Supporting Information}

The Supporting Information is available free of charge on the ACS Publications website at DOI: 10.1021/acsenergylett. $8 \mathrm{~b} 00465$.
Experimental section, $J-V$ curves before and after IS measurements at different times to test degradation, comparison of the voltage scan at fixed illumination, and an illumination scan at $V_{\text {oc }}$ with a different number of frequencies measured and their corresponding $J-V$ curves before and after the measurements (PDF)

\section{AUTHOR INFORMATION}

\section{Corresponding Authors}

*E-mail: thierry.pauporte@chimie-paristech.fr (T.P.).

*E-mail: sero@uji.es (I.M.-S.).

ORCID

Thierry Pauporté: 0000-0001-5906-8075

Iván Mora-Seró: 0000-0003-2508-0994

Notes

Views expressed in this Viewpoint are those of the authors and not necessarily the views of the ACS.

The authors declare no competing financial interest.

\section{ACKNOWLEDGMENTS}

T.P. acknowledges COST Action MP1307 StableNextSol for supporting his visit at INAM (Short Term Scientific Mission $\mathrm{n}^{\circ}$ 38419). This work was partially supported by the European Research Council (ERC) via a Consolidator Grant (724424 No-LIMIT).

\section{REFERENCES}

(1) Yang, W. S.; Park, B.-W.; Jung, E. H.; Jeon, N. J.; Kim, Y. C.; Lee, D. U.; Shin, S. S.; Seo, J.; Kim, E. K.; Noh, J. H.; Seok, S. I. Iodide Management in Formamidinium-Lead-Halide-Based Perovskite Layers for Efficient Solar Cells. Science 2017, 356, 1376-1379.

(2) Fakharuddin, A.; Schmidt-Mende, L.; Garcia-Belmonte, G.; Jose, R.; Mora-Sero, I. Interfaces in Perovskite Solar Cells. Adv. Energy Mater. 2017, 7, 1700623.

(3) Im, J.-H.; Lee, C.-R.; Lee, J.-W.; Park, S.-W.; Park, N.-G. 6.5\% Efficient Perovskite Quantum-Dot-Sensitized Solar Cell. Nanoscale 2011, 3, 4088-4093.

(4) Kojima, A.; Teshima, K.; Shirai, Y.; Miyasaka, T. Organometal Halide Perovskites as Visible-Light Sensitizers for Photovoltaic Cells. J. Am. Chem. Soc. 2009, 131, 6050-6051.

(5) Christians, J. A.; Manser, J. S.; Kamat, P. V. Best Practices in Perovskite Solar Cell Efficiency Measurements. Avoiding the Error of Making Bad Cells Look Good. J. Phys. Chem. Lett. 2015, 6, 852-857.

(6) Fabregat-Santiago, F.; Garcia-Belmonte, G.; Mora-Seró, I.; Bisquert, J. Characterization of Nanostructured Hybrid and Organic Solar Cells by Impedance Spectroscopy. Phys. Chem. Chem. Phys. 2011, 13, 9083-9118. 
(7) Anaya, M.; Zhang, W.; Hames, B. C.; Li, Y.; Fabregat-Santiago, F.; Calvo, M. E.; Snaith, H. J.; Miguez, H.; Mora-Sero, I. Electron Injection and Scaffold Effects in Perovskite Solar Cells. J. Mater. Chem. C 2017, 5, 634-644.

(8) Zarazua, I.; Han, G.; Boix, P. P.; Mhaisalkar, S.; FabregatSantiago, F.; Mora-Seró, I.; Bisquert, J.; Garcia-Belmonte, G. Surface Recombination and Collection Efficiency in Perovskite Solar Cells from Impedance Analysis. J. Phys. Chem. Lett. 2016, 7, 5105-5113.

(9) Zarazúa, I.; Sidhik, S.; Lopéz-Luke, T.; Esparza, D.; De la Rosa, E.; Reyes-Gomez, J.; Mora-Seró, I.; Garcia-Belmonte, G. Operating Mechanisms of Mesoscopic Perovskite Solar Cells through Impedance Spectroscopy and J-V Modeling. J. Phys. Chem. Lett. 2017, 8, 60736079

(10) Juarez-Perez, E. J.; Wu/ler, M.; Fabregat-Santiago, F.; LakusWollny, K.; Mankel, E.; Mayer, T.; Jaegermann, W.; Mora-Sero, I. Role of the Selective Contacts in the Performance of Lead Halide Perovskite Solar Cells. J. Phys. Chem. Lett. 2014, 5, 680-685.

(11) Wang, P.; Shao, Z.; Ulfa, M.; Pauporté, T. Insights into the Hole Blocking Layer Effect on the Perovskite Solar Cell Performance and Impedance Response. J. Phys. Chem. C 2017, 121, 9131-9141.

(12) Zhang, J.; Juarez-Perez, E. J.; Mora-Sero, I.; Viana, B.; Pauporté, T. Fast and Low Temperature Growth of Electron Transport Layers for Efficient Perovskite Solar Cells. J. Mater. Chem. A 2015, 3, 49094915.

(13) Pockett, A.; Eperon, G. E.; Peltola, T.; Snaith, H. J.; Walker, A.; Peter, L. M.; Cameron, P. J. Characterization of Planar Lead Halide Perovskite Solar Cells by Impedance Spectroscopy, Open-Circuit Photovoltage Decay, and Intensity-Modulated Photovoltage/Photocurrent Spectroscopy. J. Phys. Chem. C 2015, 119, 3456-3465.

(14) Guillén, E.; Ramos, F. J.; Anta, J. A.; Ahmad, S. Elucidating Transport-Recombination Mechanisms in Perovskite Solar Cells by Small-Perturbation Techniques. J. Phys. Chem. C 2014, 118, 2291322922.

(15) Contreras, L.; Idigoras, J.; Todinova, A.; Salado, M.; Kazim, S.; Ahmad, S.; Anta, J. A. Specific Cation Interactions as the Cause of Slow Dynamics and Hysteresis in Dye and Perovskite Solar Cells: a Small-Perturbation Study. Phys. Chem. Chem. Phys. 2016, 18, 3103331042.

(16) Pascoe, A. R.; Duffy, N. W.; Scully, A. D.; Huang, F.; Cheng, Y.B. Insights into Planar $\mathrm{CH}_{3} \mathrm{NH}_{3} \mathrm{PbI}_{3}$ Perovskite Solar Cells Using Impedance Spectroscopy. J. Phys. Chem. C 2015, 119, 4444-4453.

(17) Dualeh, A.; Moehl, T.; Tétreault, N.; Teuscher, J.; Gao, P.; Nazeeruddin, M. K.; Grätzel, M. Impedance Spectroscopic Analysis of Lead Iodide Perovskite-Sensitized Solid-State Solar Cells. ACS Nano 2014, 8, 362-373.

(18) Zarazua, I.; Bisquert, J.; Garcia-Belmonte, G. Light-Induced Space-Charge Accumulation Zone as Photovoltaic Mechanism in Perovskite Solar Cells. J. Phys. Chem. Lett. 2016, 7, 525-528.

(19) Kim, H.-S.; Lee, J.-W.; Yantara, N.; Boix, P. P.; Kulkarni, S. A.; Mhaisalkar, S.; Gratzel, M.; Park, N.-G. High Efficiency Solid-State Sensitized Solar Cell-Based on Submicrometer Rutile TiO2 Nanorod and $\mathrm{CH}_{3} \mathrm{NH}_{3} \mathrm{PbI}_{3}$ Perovskite Sensitizer. Nano Lett. 2013, 13, 24122417.

(20) Dharani, S.; Dewi, H. A.; Prabhakar, R. R.; Baikie, T.; Shi, C.; Yonghua, D.; Mathews, N.; Boix, P. P.; Mhaisalkar, S. G. Incorporation of $\mathrm{Cl}$ into Sequentially Deposited Lead Halide Perovskite Films for Highly Efficient Mesoporous Solar Cells. Nanoscale 2014, 6, 1385413860 .

(21) Juarez-Perez, E. J.; Sanchez, R. S.; Badia, L.; Garcia-Belmonte, G.; Kang, Y. S.; Mora-Sero, I.; Bisquert, J. Photoinduced Giant Dielectric Constant in Lead Halide Perovskite Solar Cells. J. Phys. Chem. Lett. 2014, 5, 2390-2394.

(22) Fabregat-Santiago, F.; Kulbak, M.; Zohar, A.; Vallés-Pelarda, M.; Hodes, G.; Cahen, D.; Mora-Seró, I. Deleterious Effect of Negative Capacitance on the Performance of Halide Perovskite Solar Cells. ACS Energy Lett. 2017, 2, 2007-2013.

(23) Wang, P.; Ulfa, M.; Pauporté, T. Effects of Perovskite Monovalent Cation Composition on the High and Low Frequency
Impedance Response of Efficient Solar Cells. J. Phys. Chem. C 2018, 122, 1973-1981.

(24) Zhang, J.; Pauporté, T. Effects of Oxide Contact Layer on the Preparation and Properties of $\mathrm{CH}_{3} \mathrm{NH}_{3} \mathrm{PbI}_{3}$ for Perovskite Solar Cell Application. J. Phys. Chem. C 2015, 119, 14919-14928.

(25) Mora-Seró, I.; Garcia-Belmonte, G.; Boix, P. P.; Vázquez, M. A.; Bisquert, J. Impedance Spectroscopy Characterisation of Highly Efficient Silicon Solar Cells under Different Light Illumination Intensities. Energy Environ. Sci. 2009, 2, 678-686.

(26) Sanchez, R. S.; Gonzalez-Pedro, V.; Lee, J.-W.; Park, N.-G.; Kang, Y. S.; Mora-Sero, I.; Bisquert, J. Slow Dynamic Processes in Lead Halide Perovskite Solar Cells. Characteristic Times and Hysteresis. J. Phys. Chem. Lett. 2014, 5, 2357-2363.

(27) Saliba, M.; Matsui, T.; Seo, J.-Y.; Domanski, K.; Correa-Baena, J.-P.; Nazeeruddin, M. K.; Zakeeruddin, S. M.; Tress, W.; Abate, A.; Hagfeldt, A.; et al. Cesium-Containing Triple Cation Perovskite Solar Cells: Improved Stability, Reproducibility and High Efficiency. Energy Environ. Sci. 2016, 9, 1989-1997.

(28) Tsai, H.; Nie, W.; Blancon, J.-C.; Stoumpos, C. C.; Asadpour, R.; Harutyunyan, B.; Neukirch, A. J.; Verduzco, R.; Crochet, J. J.; Tretiak, S.; et al. High-Efficiency Two-Dimensional RuddlesdenPopper Perovskite Solar Cells. Nature 2016, 536, 312-316.

(29) Domanski, K.; Alharbi, E. A.; Hagfeldt, A.; Grätzel, M.; Tress, W. Systematic Investigation of the Impact of Operation Conditions on the Degradation Behaviour of Perovskite Solar Cells. Nature Energy 2018, 3, 61-67.

(30) Saliba, M. Perovskite Solar Cells Must Come of Age. Science 2018, 359, 388.

(31) Murugadoss, G.; et al. Light Stability Tests of Methylammonium and Formamidinium $\mathrm{Pb}$-Halide Perovskites for Solar Cell Applications. Jpn. J. Appl. Phys. 2015, 54, 08KF08.

(32) Gottesman, R.; Zaban, A. Perovskites for Photovoltaics in the Spotlight: Photoinduced Physical Changes and Their Implications. Acc. Chem. Res. 2016, 49, 320-329.

(33) Tress, W.; Yavari, M.; Domanski, K.; Yadav, P.; Niesen, B.; Correa Baena, J. P.; Hagfeldt, A.; Graetzel, M. Interpretation and Evolution of Open-Circuit Voltage, Recombination, Ideality Factor and Subgap Defect States During Reversible Light-Soaking and Irreversible Degradation of Perovskite Solar Cells. Energy Environ. Sci. 2018, 11, 151-165. 